\title{
Line Integral Convolution for Visualization of Fiber Tract Maps from DTI
}

\author{
T. McGraw ${ }^{1}$, B.C. Vemuri ${ }^{1}$, Z. Wang ${ }^{1}$, Y. Chen $^{2}$, M. Rao ${ }^{2}$, and T. Mareci ${ }^{3}$ \\ 1 Dept. of CISE, University of Florida, Gainesville, Fl. 32611 \\ 2 Dept. of Mathematics, University of Florida, Gainesville, Fl. 32611 \\ 3 Dept. of Biochemistry, University of Florida, Gainesville, Fl. 32610
}

\begin{abstract}
Diffusion tensor imaging (DTI) can provide the fundamental information required for viewing structural connectivity. However, robust and accurate acquisition and processing algorithms are needed to accurately map the nerve connectivity. In this paper, we present a novel algorithm for extracting and visualizing the fiber tracts in the CNS specifically, the spinal cord. The automatic fiber tract mapping problem will be solved in two phases, namely a data smoothing phase and a fiber tract mapping phase. In the former, smoothing is achieved via a weighted $\mathrm{TV}$-norm minimization which strives to smooth while retaining all relevant detail. For the fiber tract mapping, a smooth $3 \mathrm{D}$ vector field indicating the dominant anisotropic direction at each spatial location is computed from the smoothed data. Visualization of the fiber tracts is achieved by adapting a known Computer Graphics technique called the line integral convolution, which has the advantage of being able to cope with singularities in the vector field and is a resolution independent way of visualizing the $3 \mathrm{D}$ vector field corresponding to the dominant eigen vectors of the diffusion tensor field. Examples are presented to depict the performance of the visualization scheme on three DT-MR data sets, one from a normal and another from an injured rat spinal cord and a third from a rat brain.
\end{abstract}

\section{Introduction}

Fundamental advances in understanding living biological systems require detailed knowledge of structural and functional organization. Recently MR imaging has been used to study the structural connectivity within whole living organisms. The MR measurement of water translational self-diffusion provides a method that can be used to study structural connectivity with ubiquitous indigenous material, water. In highly organized nervous tissue, like white matter, diffusion anisotropy can be used to visualize fiber tracts. Recently MR measurements have been developed to measure the tensor of diffusion. The development of diffusion tensor acquisition, processing, and analysis methods provides the framework for creating fiber tract maps based on this complete diffusion tensor analysis 9 10,12]13.

For automated fiber tract mapping, prior to estimating the diffusion tensor, the raw data must be smoothed while preserving relevant detail. The raw data 
in this context consists of seven directional images acquired for varying magnetic field strengths. Note that at least seven values at each $3 \mathrm{D}$ grid point in the data domain are required to estimate the six unknowns in the symmetric, rank 2 tensor and one scale parameter. The data smoothing or de-noising can be formulated using variational principles which in turn require solutions to partial differential equations (PDEs). We will limit ourselves to vector-valued image smoothing and refer the reader to 214] for scalar-valued image smoothing techniques. Whitaker and Gerig [23 introduced anisotropic vector-valued diffusion which was a direct extension of the work by Perona and Malik [15] to vector-valued images. In [19] Sapiro et.al., introduced a selective smoothing technique based on the Riemanian metric of the underlying manifold of the vector-valued function. This was applied to restoration of color images. A very general flow called the Beltrami flow was introduced in Kimmel et. al., [1] and was shown that most flow-based smoothing schemes may be viewed as special cases in their framework. A generalization of the total variation (TV) norm to handle vector-valued image smoothing was presented in Blomgren and Chan [2]. For more on other flow-based smoothing methods, we refer the reader to a survey by Weickert [21] and also [4.

More recently, Poupon et.al., [17] developed a Bayesian formulation of the fiber tract mapping problem. Prior to mapping the fibers, they use robust regression to estimate the diffusion tensor from the vector valued image data. Note that no image selective smoothing is performed in their work prior to application of the robust regression for estimating the diffusion tensors.

Previously, Westin, et. al. [22] presented a smoothing method applied strictly in the tensor domain, after diffusion tensors had been computed from noisy raw data. We propose a novel and efficient weighted TV norm based image smoothing scheme where in the raw image data (one image for each of the 7 directions) $\mathbf{S}$ is smoothed using a PDE which is obtained as a consequence of a weighted TV norm minimization defined for vector valued functions. The selective term in our work is based on the eigen values of a diffusion tensor $\mathbf{D}$ that can be computed initially from the raw image data using the relationship $\mathbf{S}=S_{0} \exp \left(-\sum_{i j} b_{i j} D_{i j}\right)$, where, $\mathbf{S}$ is the vector of signal/image measurements taken along seven directions $X, Y, Z, X Y, Y Z, X Z, X Y Z, S_{0}$ is a constant, $b_{i j}$ is the magnetic field strength (which is a constant for a given direction) and $D_{i j}$ are the entries of the $(3,3)$ matrix representing the diffusion tensor measuring the diffusion of water inside the body being imaged. The selective term in this case $g(s)=1 /(1+s)$ where $s=F A$ is the fractional anisotropy defined as 1 . This selection criteria preserves the dominant anisotropic direction while smoothing the rest of the data. Note that since we are only interested in the fiber tracts which correspond to the streamlines of the dominant anisotropic direction, it is apt to choose such a selective term as opposed to one that preserves edges in signal intensity as was done in [14].

Given the dominant eigen vector field of the diffusion tensor in 3D, tracking the fibers (space curves) is basically equivalent to finding the stream lines/integral curves in 3D of this vector field. Finding integral curves of vector fields is a well researched problem in the field of Fluid Mechanics [8]. The simplest solution 
would be to numerically integrate the given vector field using a stable numerical integration scheme such as a fourth order Runge-Kutta integrator [18. The problem with streamline finding methods is that there is no clean way to deal with singularities in the vector field. We propose to use a technique from Computer Graphics called the line integral convolution (LIC) which involves convolving a known texture with the vector field such that the result creates the perception of the flow/streamlines in the known texture. This may be achieved on a plane or on any desired manifold/surface. This scheme is resolution independent and can easily cope with singularities in vector fields. Previously, Chaing, et. al. [7] have used LIC to render diffusion tensor images of the myocardium. We present the visualization of reconstructed $3 \mathrm{D}$ vector fields rendered using the LIC technique. Images corresponding to the dominant eigen vectors of the tensor field for a normal and an injured rat spinal cord, as well as a rat brain are presented.

\section{Image De-noising and Diffusion Tensor Computation}

Smoothing the raw vector valued image data is posed as a variational principle involving a first order smoothness constraint on the solution to the smoothing problem. Let $\hat{\mathbf{S}}(\mathbf{X})$ be the vector valued image that we want to smooth where, $\mathbf{X}=(x, y, z)$ and let $\mathbf{S}(X)$ be the unknown smooth approximation of the data that we want to estimate. We propose a weighted TV-norm minimization for smoothing the vector valued image $\mathbf{S}$. The variational principle for estimating a smooth $\mathbf{S}(\mathbf{X})$ is given by

$$
\min _{\mathbf{S}} \mathcal{E}(\mathbf{S})=\int_{\Omega}\left[\mathbf{g}\left(\lambda_{+}, \lambda_{-}\right) \sum_{\mathbf{i}=1}^{\mathbf{7}}\left|\nabla \mathbf{S}_{\mathbf{i}}\right|+\frac{\mu}{\mathbf{2}} \sum_{\mathbf{i}=1}^{\mathbf{7}}\left|\mathbf{S}_{\mathbf{i}}-\hat{\mathbf{S}}_{\mathbf{i}}\right|^{\mathbf{2}}\right] \mathbf{d} \mathbf{x}
$$

where, $\Omega$ is the image domain and $\mu$ is a regularization factor. The first term here is the regularization constraint on the solution to have a certain degree of smoothness. The second term in the variational principle makes the solution faithful to the data to a certain degree. The gradient descent of the above minimization is given by

$$
\begin{aligned}
& \frac{\partial \mathrm{S}_{i}}{\partial t}=\operatorname{div}\left(\frac{g\left(\lambda_{+}, \lambda_{-}\right) \nabla \mathrm{S}_{i}}{\left\|\nabla S_{i}\right\|}\right)-\mu\left(\mathrm{S}_{i}-\hat{\mathrm{S}}_{i}\right) \quad i=1, \ldots, 7 \\
& \left.\frac{\partial S_{i}}{\partial n}\right|_{\partial \Omega \times \Re+}=0 \text { and } \mathbf{S}(\mathbf{x}, t=0)=\hat{\mathbf{S}}(\mathbf{x})
\end{aligned}
$$

Note that we can prove the convergence of this PDE to the true solution without invoking viscosity methods. Existence and uniqueness issues of a solution have been worked out as well however, such proofs are beyond the scope of this paper. The above nonlinear PDE is solved using an efficient and stable numerical scheme namely, the Crank-Nicholson scheme 16.

\subsection{Visualizing the Stream Lines}

Once the diffusion tensor has been robustly estimated, the principal diffusion direction can be calculated by finding the eigen vector corresponding to the 
dominant eigen value of this tensor. The fiber tracts may be mapped by visualizing the streamlines through the field of eigen vectors.

LIC is a texture-based vector field visualization method suggested by Cabral .et al. in 3. The technique generates intensity values by convolving a noise texture with a curvilinear kernel aligned with the streamline through each pixel, such as by:

$$
I\left(x_{0}\right)=\int_{s_{0}-L}^{s_{0}+L} T(\sigma(s)) k\left(s_{0}-s\right) d s
$$

where $I\left(x_{0}\right)$ is the intensity of the LIC texture at pixel $x_{0}, k$ is a filter kernel of width $2 L, T$ is the input noise texture, and $\sigma$ is the streamline through point $x_{0}$. The streamline, $\sigma$ can be found by numerical integration, given the discrete field of eigen vectors.

The result is a texture with highly correlated values between nearby pixels on the same streamline, and contrasting values for pixels not sharing a streamline. In our case, an FA value below a certain threshold can be a stopping criterion for the integration since the diffusion field ceases to have a principal direction for low FA values.

Stalling and Hege 20] achieve significant computational savings by leveraging the correlation between adjacent points on the same streamline. For a constant valued kernel, $k$, the intensity value at $I(\sigma(s+d s))$ can be quickly estimated by $I(\sigma(s))+\epsilon$, where $\epsilon$ is a small error term which can be easily computed.

\section{Experimental Results}

In this section, we present three sets of experiments, two on rat spinal cord data sets and one on a rat brain.

In all of the experiments, we first smooth the seven 3D directional images using the novel selective smoothing technique outlined in section 2. Following this, the diffusion tensor is estimated from the smoothed data using a standard least squares technique. The fractional anisotropy and the direction cosines of the eigen vector corresponding to the dominant eigen value are computed. The latter image depicts the standard axis $(X, Y, Z)$ toward which direction of diffusion in the data is dominant and the dominant eigen value corresponds to the magnitude of this dominant diffusion direction. Each direction is represented by a corresponding grey value. This coding will indicate the standard direction (X, $\mathrm{Y}$ or $\mathrm{Z}$ ) along which the dominant eigen vector has the strongest component. Images obtained as a result of these computations from raw data, smoothed data using a competing smoothing method outlined in Parker et.al., [14] and smoothed data using our proposed method are depicted for the three data sets. In addition, estimated fiber tracts from the smoothed data using the proposed LIC method are depicted for the data sets. The results of smoothing for the three examples are shown in Figure 1, which is organized as follows: first column contains images computed from raw (noisy) data, second column contains images computed using methods in [14] and third column contains computed images using the proposed image smoothing technique. Note the superior performance 


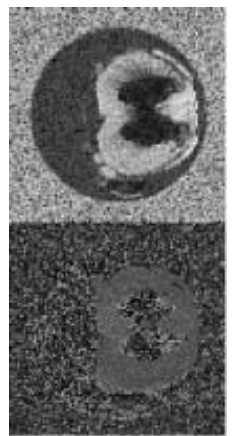

(a)

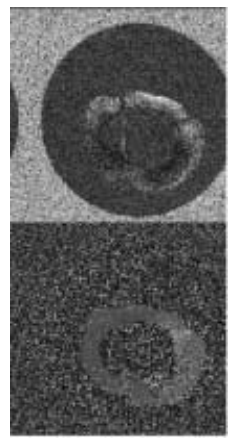

(d)

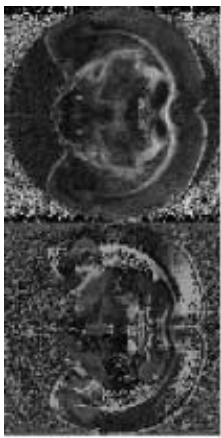

$(\mathrm{g})$

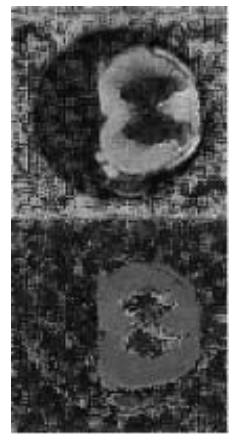

(b)

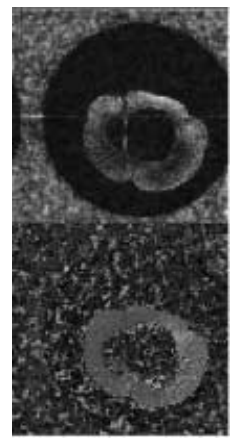

(e)

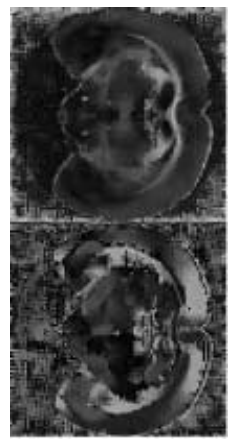

(h)

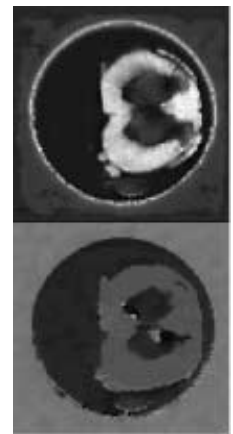

(c)

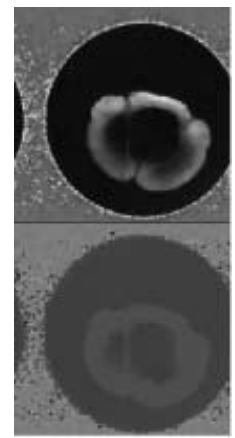

(f)

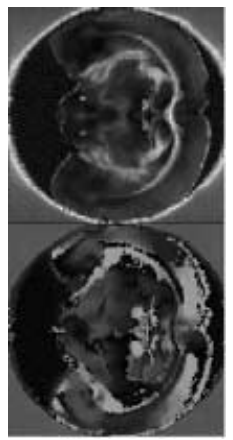

(i)

Fig. 1. Top and bottom panel of (a), (b) \& (c): FA and direction cosines for normal spinal cord. (a) Results computed from raw data. (b) Results computed using Parker's method. (c) Results from the proposed smoothing; (d),(e) \& (f): similar results for injured cord; (g), (h), (i): similar results for rat brain. 


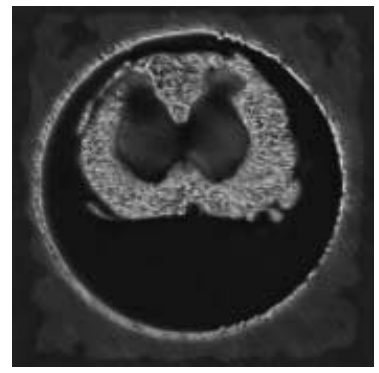

(a)

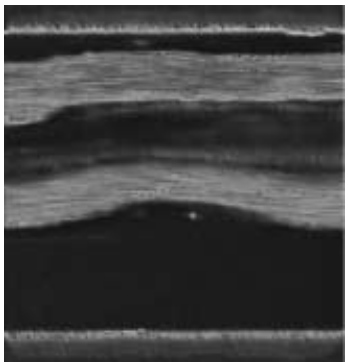

(b)

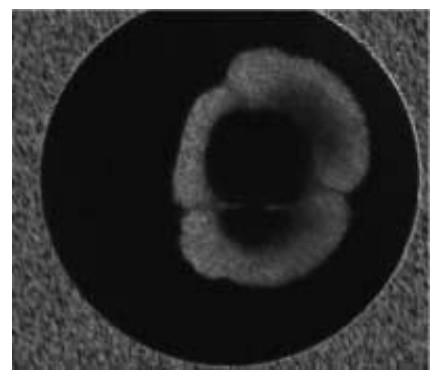

(c)

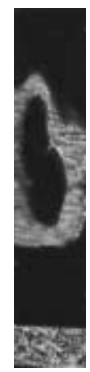

(d)

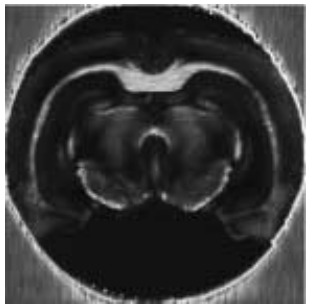

(e)

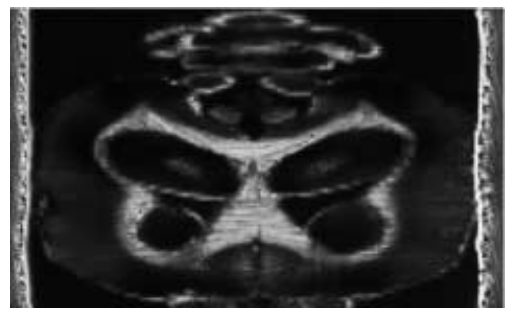

(f)

Fig. 2. Fiber tracts computed from smoothed data. (a) Normal spinal cord axial slice, and (b) sagittal slice; (c) Injured spinal cord axial slice, and (d) sagittal slice; (e) Rat brain coronal slice, and (f) axial slice.

of the proposed smoothing scheme in comparison to the method in Parker et. al., 14.

Figure 2 depicts the computed fiber tracts for the three reconstructed data sets. The intensity of the LIC texture has be modulated with the FA image to emphasize the most anisotropic region of each image. The top row shows the LIC fiber map in two perpendicular planes for the normal rat spinal cord. These fiber tracts are supposed to run along the length of the spinal cord in the white 
matter which is exactly what the LIC reveals. For the axial image, the fibers are perpendicular to the image plane, so LIC in this case generates uncorrelated noise. In the sagittal view, however, the fibers lie in the image plane, and are visible as an oriented texture.

In the middle row, LIC is applied to the injured spinal cord data. As evident in the sagittal slice, the injury has caused a large cavity down the length of the spine and there are no fibers in this region.

The bottom row shows the results for the brain. As expected, fiber tracts are clearly visible in the region of the corpus collosum. In all three cases, the visual quality of the fiber tracts is satisfactory.

In the above presented results, it should be noted is that we have demonstrated a proof of concept for the proposed data smoothing and fiber tract mapping algorithms in the case of the normal and injured rat spinal cords, and normal brain respectively. The quality of results obtained is reasonably satisfactory for visual inspection purposes but quantitative validation needs to be performed and will be the focus of our future efforts.

\section{Conclusions}

In this paper, we presented a new weighted TV-norm minimization formulation for smoothing vector-valued data specifically tuned to computation of smooth diffusion tensor MR images. The smoothed vector valued data was then used to compute a diffusion tensor image using standard least squares technique. Fiber tracts were estimated using the dominant eigen vector field obtained from the diffusion tensor image. Finally, results of fiber tract mapping of a normal and an injured rat spinal cord, and a rat brain were depicted using LIC. The fiber tracts are quite accurate when inspected visually. However, quantitative validation of the computed fiber tracts is essential and will be the focus of our future efforts.

\section{Acknowledgement}

This research was funded in part by the NIH grant RO1-NS42075.

\section{References}

1. P. J. Basser and C. Pierpaoli "Microstructural and Physiological Features of Tissue Elucidated by Quantitative-Diffusion-Tensor MRI," J. Magn. Reson. B 110, 209219 (1996)

2. P. Blomgren and T. F. Chan," Color TV: Total Variation Methods for Restration of Vector-Valued Images," IEEE Transaction on Image Processing, Vol. 7, no. 3, pp. 304-309, March, 1998.

3. B. Cabral and L. Leedom,"Imaging Vector Fields Using Line Integral Convolution," Proc. of SIGGRAPH' '93, pp. 263-272, 1993.

4. V. Caselles, J. M. Morel, G. Sapiro and A. Tannenbaum,IEEE Trans. on IP, special issue on PDEs and geometry-driven diffusion in image processing and analysis, Vol 7, No. 3, 1998. 
5. T. Chan and P. Mulet, "On the Convergence of the Lagged Diffusivity Fixed Point Method in Total Variation Image Restoration," September 1997, CAM TR-97-46.

6. T. Chan and J. Shen, "Variational restoration of non-flat image features: model and algorithm," Technical Report, CAM-TR 99-02, UCLA, 1999.

7. P.-J. Chiang, B. Davis and E. Hsu, "Line-Integral Convolution Reconstruction of Tissue Fiber Architecture Obtained by MR Diffusion Tensor Imaging," BMES Annual Meeting, 2000.

8. A. Chorin, Computational Fluid Mechanics, Selected papers, Academic Press, 1989.

9. T. E. Conturo, et.al., "Tracking neuronal fiber pathways in the living human brain," Proc. Natl. Acad. Sci. USA 96, 10422-10427 (1999)

10. D. K. Jones, A. Simmons, S. C. R. Williams and M. A. Horsfield, "Non-invasive assessment of axonal fiber connectivity in the human brain via diffusion tensor MRI," Magn. Reson. Med., 42, 37-41 (1999).

11. R.Kimmel, N.Sochen, and R.Malladi, "Images as embedding maps and minimal surfaces:movies, color and volumetric medical images," in Proc. of the IEEE Conf. on CVPR, June 1997, pp. 350-355.

12. N. Makris, et.al., "Morphometry of in vivo human white matter association pathways with diffusion-weighted magnetic resonance imaging," Ann. Neurol., 42, 951962 (1999).

13. S. Mori, B. J. Crain, V. P. Chacko and P. C. M. van Zijl "Three-dimensional tracking of axonal projections in the brain by magnetic resonance imaging," Ann. Neurol., 45, 265-269 (1999)

14. G.J. M. Parker, J. A. Schnabel, M. R. Symms, D. J. Werring and G. J. Baker, "Nonlinaer smoothing for reduction of systematic and random errors in diffusion tensor imaging," Magn. Reson. Imag., 11, 702-710, 2000.

15. P.Perona and J.Malik, "Scale-space and edge detection using anisotropic diffusion," IEEE TPAMI, vol. 12, no. 7, pp. 629-639, 1990.

16. L. Lapidus and G. F. Pinder, Numerical solution of partial differential equations in science and engineering, John Wiley and Sons, 1982.

17. C. Poupon, C. A. Clark et.al., "Regularization of diffusion-based direction maps for the tracking of brain white matter fascicles," NeuroImage, 12, 184-195, 2000.

18. W.H.Press, B.P.Flannery, S.A.Teukolsky and W.T.Vetterling, [1992], Numerical Recipes in C: The Art of Scientific Computing. Cambridge University Press.

19. G.Sapiro and D.L. Ringach, "Anisotropic diffusion of multivalued images with applications to color filtering," IEEE TIP, vol. 5, pp. 1582-1586, 1996.

20. D.Stalling and H.C. Hege, "Fast and Resolution Independent Line Integral Convolution," Proc. of SIGGRAPH '95, pp. 249-256, 1995.

21. J.Weickert, "A review of nonlinear diffusion filtering," in Scale-space theory in computer vision,, (Eds.) B. ter Haar Romney et.al. 1997, vol. 1252, pp. 3-28, Springer-Verlag.

22. C.-F. Westin, S.E. Maier, B. Khidhir, P. Everett, F.A. Jolesz and R. Kikinis, "Image Processing for Diffusion Tensor Magnetic Resonance Imaging," in Proceedings of MICCAI' '99, pp. 441-452, 1999

23. R. Whitaker and G. Gerig, "Vector-valued diffusions," in Geometry-driven Diffusions in Computer Vision, B. Romney et.al., (Eds.), Kluwer, 1994. 